University of Nebraska - Lincoln

DigitalCommons@University of Nebraska - Lincoln

U.S. Environmental Protection Agency Papers

U.S. Environmental Protection Agency

2011

Current and Future Needs for Developmental Toxicity Testing

Susan L. Makris

U.S. Environmental Protection Agency, makris.susan@epa.gov

James H. Kim

ILSI Health and Environmental Sciences Institute

Amy Ellis

U.S. Food and Drug Administration

Willem Faber

Willem Faber Toxicology Consulting

Wafa Harrouk

U.S. Food and Drug Administration

See next page for additional authors

Follow this and additional works at: https://digitalcommons.unl.edu/usepapapers

Part of the Civil and Environmental Engineering Commons

Makris, Susan L.; Kim, James H.; Ellis, Amy; Faber, Willem; Harrouk, Wafa; Lewis, Joseph M.; Paule, Merle G.; Seed, Jennifer; Tassinari, Melissa; and Tyl, Rochelle, "Current and Future Needs for Developmental Toxicity Testing" (2011). U.S. Environmental Protection Agency Papers. 88.

https://digitalcommons.unl.edu/usepapapers/88

This Article is brought to you for free and open access by the U.S. Environmental Protection Agency at DigitalCommons@University of Nebraska - Lincoln. It has been accepted for inclusion in U.S. Environmental Protection Agency Papers by an authorized administrator of DigitalCommons@University of Nebraska - Lincoln. 
Authors

Susan L. Makris, James H. Kim, Amy Ellis, Willem Faber, Wafa Harrouk, Joseph M. Lewis, Merle G. Paule, Jennifer Seed, Melissa Tassinari, and Rochelle Tyl

This article is available at DigitalCommons@University of Nebraska - Lincoln: https://digitalcommons.unl.edu/ 


\title{
Review Article \\ Current and Future Needs for Developmental Toxicity Testing
}

\author{
Susan L. Makris, ${ }^{1 *}$ James H. Kim, ${ }^{2}$ Amy Ellis, ${ }^{3}$ Willem Faber, ${ }^{4}$ Wafa Harrouk, ${ }^{3}$ \\ Joseph M. Lewis, ${ }^{5}$ Merle G. Paule, ${ }^{6}$ Jennifer Seed, ${ }^{7}$ Melissa Tassinari, ${ }^{8}$ and Rochelle Tyl ${ }^{9}$ \\ ${ }^{1}$ U.S. Environmental Protection Agency, National Center for Environmental Assessment, Office of Research and Development, \\ Washington, District of Columbia \\ ${ }^{2}$ ILSI Health and Environmental Sciences Institute, Washington, District of Columbia \\ ${ }^{3}$ U.S. Food and Drug Administration, Center for Drug Evaluation and Research, Silver Spring, Maryland \\ ${ }^{4}$ Willem Faber Toxicology Consulting, Victor, New York \\ ${ }^{5}$ E.I. du Pont de Nemours and Co., Wilmington, Delaware \\ ${ }^{6}$ U.S. Food and Drug Administration, National Center for Toxicological Research, Jefferson, Arkansas \\ ${ }^{7}$ U.S. Environmental Protection Agency, Office of Pollution Prevention and Toxics, Washington, District of Columbia \\ ${ }^{8}$ Pfizer, Inc., Groton, Connecticut \\ ${ }^{9}$ RTI International, Research Triangle Park, North Carolina
}

A review is presented of the use of developmental toxicity testing in the United States and international regulatory assessment of human health risks associated with exposures to pharmaceuticals (human and veterinary), chemicals (agricultural, industrial, and environmental), food additives, cosmetics, and consumer products. Developmental toxicology data are used for prioritization and screening of pharmaceuticals and chemicals, for evaluating and labeling of pharmaceuticals, and for characterizing hazards and risk of exposures to industrial and environmental chemicals. The in vivo study designs utilized in hazard characterization and dose-response assessment for developmental outcomes have not changed substantially over the past 30 years and have served the process well. Now there are opportunities to incorporate new technologies and approaches to testing into the existing assessment paradigm, or to apply innovative approaches to various aspects of risk assessment. Developmental toxicology testing can be enhanced by the refinement or replacement of traditional in vivo protocols, including through the use of in vitro assays, studies conducted in alternative nonmammalian species, the application of new technologies, and the use of in silico models. Potential benefits to the current regulatory process include the ability to screen large numbers of chemicals quickly, with the commitment of fewer resources than traditional toxicology studies, and to refine the risk assessment process through an enhanced understanding of the mechanisms of developmental toxicity and their relevance to potential human risk. As the testing paradigm evolves, the ability to use developmental toxicology data to meet diverse critical regulatory needs must be retained. Birth Defects Res (Part B) 92:384-394, 2011. $\quad$ (C) 2011 Wiley Periodicals, Inc.

\section{Key words: developmental toxicology testing; pharmaceuticals; environ- mental chemicals; food additives; cosmetics; consumer products; risk assessment}

\section{INTRODUCTION}

The assessment of developmental toxicology data is a critical aspect of hazard evaluation for pharmaceuticals and environmental chemicals. The International Life Sciences Institute (ILSI) Health and Environmental Sciences Institute (HESI) Developmental and Reproductive Toxicology Technical Committee initiated a project to conduct a comprehensive critical review of the state of the art for developmental toxicity testing for safety assessment, and to explore future directions. A public Workshop was held on April 29-30, 2009 in Washington, DC to explore this issue in greater depth.

In this article, we describe the current status of developmental toxicity testing within a variety of programs and discuss other considerations that are of significant influence on the future state of the science. This serves as an introduction to topic papers that provide a detailed critical evaluation of current developmental toxicity testing strategies (Carney et al., 2011), define possible refinements of testing strategies to

*Correspondence to: Susan L. Makris, U.S. EPA/ORD/NCEA-W (Mailcode 8623P), 1200 Pennsylvania Avenue, NW, Washington, DC 20460. E-mail: makris.susan@epa.gov

Melissa Tassinari's Current Address is U.S. Food and Drug Administration, Center for Drug Evaluation and Research, Silver Spring, Maryland. Received 12 May 2011; Accepted 22 July 2011

Published online in Wiley Online Library (wileyonlinelibrary.com) DOI: $10.1002 /$ bdrb.20335 
maximize relevance for human safety assessment (Brannan et al., 2011), and discuss new approaches and technologies for developmental toxicity testing for safety assessment (Knudsen et al., 2011).

Developmental toxicity is defined as the study of adverse effects on the developing organism that may result from exposure to either parent before conception, during prenatal development, or postnatally to the time of sexual maturation (U.S. EPA, 1991; OECD, 2008a). Adverse developmental effects may be detected at any point in the lifespan of the organism. The major manifestations of developmental toxicity are death, structural abnormality, altered growth, and functional deficit. Framed by this context, discussion of developmental toxicity testing is obligated to address studies that include exposures throughout any stage of development, whether prenatal or postnatal. Thus, not only traditional prenatal developmental toxicity studies but also reproductive toxicity studies (which include exposure to pregnant and lactating dams, as well as to neonatal and juvenile subjects), or studies that assess functional outcomes following exposures during critical periods of organ system development such as the developmental neurotoxicity study, are considered (OECD, 2007a). Although standardized testing protocols (i.e. test guidelines) are often treated as if they are distinct entities, they in fact represent a continuum of exposure and testing during early life stages, through growth and maturation, and across stages of primary reproductive function in adults. Study designs for guideline developmental and reproductive toxicity studies used for screening and testing pharmaceuticals, industrial chemicals, and pesticides are discussed and diagrammed in Tyl (2010) and Makris (2010).

There is an increasing recognition of the possibility of profound effects of genetic allelic diversity and of nonchemical environmental factors (e.g. food, crowding, isolation, mental and physical stimulation) on developmental and reproductive outcomes. However, past and current practices in study conduct have intentionally used outbred (40 generation) or inbred (brother-sister matings) laboratory species, with care to minimize genetic drift and founder effects in small colonies, to keep the genetic make up of the control and various treatment groups the same. Study environment (temperature, relative humidity, feed, bedding, water source, caging, etc.) is also maintained identically among all groups to remove that source of variability. Thus, typical animal test systems may not fully address the interactions between/among the test chemical, the genome of the test system, and the environment that can contribute to risk in the heterogeneous human population. Additionally, test substance administration to experimental animals is often far greater than human doses (particularly for environmental chemicals), and further, chemicals are generally administered individually to test subjects in experimental testing for regulatory purposes, while typical exposures in humans are to chemical mixtures.

\section{CURRENT USE OF DEVELOPMENTAL TOXICITY DATA UNDER VARIOUS REGULATORY PROGRAMS}

The following brief description of the primary uses of developmental toxicity data illustrates both similarities and differences in data requirements and approaches across various regulatory agencies. Some chemicals, generally due to multiple applications or uses, may fall under one or more of these categories simultaneously. For both pharmaceuticals and environmental chemicals, the US regulatory perspective is primarily highlighted; however, parallel international regulatory programs are acknowledged, and to some extent addressed in detail.

\section{Pharmaceutical (Drugs, Biologics, and Medical Devices)}

Pharmaceuticals for human use must undergo reproduction toxicity testing for the purposes of hazard identification and to aid in the evaluation of risk/benefit to women of childbearing potential who may be treated with the compound. The guidelines for testing pharmaceuticals regulated by the US FDA and the European Medicines Agency are delineated in $\mathrm{ICH}$ document S5(R2) (ICH, 2005). In the United States, reproduction and developmental toxicity data are also needed to label drug products according to Code of Federal Regulations (CFR 201.57). For most compounds, the drug's label includes information regarding any effects on fertility, embryo-fetal toxicity, teratogenic potential, and peri/postnatal development.

\section{Veterinary Pharmaceuticals}

The approval process of pharmaceuticals for veterinary use considers not only the safety of the animals that would receive the drug directly but also of humans who might consume products from a treated animal (e.g. milk, meat). To be legally marketed in the United States, new animal drug products must be approved under a NADA (New Animal Drug Application) by the FDA Center for Veterinary Medicine (CVM). Legal requirements for NADAs are delineated in 21 CFR 514. Labeling requirements for prescription veterinary drugs can be found in 21 CFR 201.105. There are several guidance documents for industry that are available from FDA/ CVM (CVM, 2006) and the International Cooperation on Harmonisation of Technical Requirements for Registration of Veterinary Medical Products (VICH) regarding reproductive and developmental toxicity testing of veterinary pharmaceuticals that is needed for approval. Target Animal Safety for Veterinary Pharmaceutical Products (VICH GL43) discusses reproduction toxicity studies that are required for systemically absorbed pharmaceuticals intended for breeding animals (VICH, 2008). These studies are to be conducted in the species for which the drug is intended and usually are not extended past the postnatal period unless there are specific concerns based on the known pharmacological and/or toxicological properties of the test article or its class. The documentsStudies to Evaluate the Safety of Residues of Veterinary Drugs in Human Food: Reproductive Toxicity Testing (VICH GL22) and Studies to Evaluate the Safety of Residues of Veterinary Drugs in Human Food: Developmental Toxicity Testing (VICH GL32) cover veterinary pharmaceuticals that may be present in residual amounts in animal products intended for human food (VICH, 2004a,b). These studies are generally conducted in rats and a multigeneration protocol is standard. 


\section{Chemical (Agricultural, Industrial, and Environmental)}

The chemical industry is one of the most highly regulated industries in the United States. This is for good reason since agricultural and chemical exposures are not typically intended or intentional, and ubiquitous and varied uses of these substances creates a wide variety of exposure scenarios. The Toxic Substances Control Act (TSCA) is the central law governing industrial chemicals. This law enables the EPA to screen and regulate new chemicals as they are coming on to the market, as well as to evaluate existing chemicals. The Federal Insecticide, Fungicide, and Rodenticide Act (FIFRA) is the primary law governing agricultural chemicals in the United States In accordance with this statute, pesticide products must be registered with EPA before manufacture, transport, or sale. Under FIFRA, chemicals are developed based on their biological activity (i.e. pesticides), whereas under TSCA, chemicals are developed based on their physical and chemical properties (e.g. paints, adhesives, films, cleansers, plasticizers); hence, the requirements for Registration under FIFRA are very different that the requirements for a pre-manufacturing notice (PMN) under TSCA. For a FIFRA registration of chemicals, the requirements are stringent and voluminous. Required studies are designed to assess toxicokinetics, environmental fate (migration into soil, ground water, air), efficacy in the target species, and toxicity into nontarget species. Toxicology studies range from acute, to subchronic, and through chronic/carcinogenicity studies, as well as including studies that address specific organ systems or biological processes, thereby providing adequate screening for systemic, reproductive, developmental, genetic and other toxicities in a broad array of test systems. For a TSCA PMN to begin production/ importation of a chemical, the requirements are relatively minimal; any toxicity data must be submitted, at least an LD50 (median lethal dose) study, the proposed use, and the proposed amount to be manufactured/imported (production volume) the first year. For some chemicals, a more extensive toxicological database may be submitted at this stage, especially for chemicals that are intended to be produced in high volume and/or for which exposure is predicted to be widespread. Under TSCA, the EPA has 90 days to accept or reject the PMN; only literature searches and/or structure-activity relationships are usually conducted by the Agency. Once the PMN has been granted under TSCA, the EPA can request (via negotiated test agreements) or require (via test rules) specific studies for specific chemicals, typically based on exposures. If the chemical, after the PMN is approved, will have additional uses, the manufacturer/importer will have to submit a Significant New Use Request, again with submission of minimum data and no required toxicology. Test guidelines that are utilized in developmental and reproductive toxicity testing for both industrial chemicals under TSCA and pesticides under FIFRA have been harmonized by the EPA Office of Prevention, Pesticides, and Toxic Substances (OPPTS). In addition, OPPTS has harmonized their toxicity testing guidelines with the multinational Organisation of Economic Co-operation and Development (OECD). The harmonized guidelines are listed in Table 1. Although OECD member countries have agreed to accept, in principle, studies conducted according to OECD guidelines, there may also be specific extant national regulations that define or modify the use of these guidelines in chemical testing (e.g. the Japanese Ministry of Agriculture, Forestry, and Fisheries [MAFF]).

\section{Food Additives}

The US FDA is the agency responsible for ensuring that the direct food additives and color additives used in food are safe for all consumers. The US FDA issued the Toxicological Principles for the Safety Assessment of Direct Food Additives and Color Additives Used in Foods, commonly referred to as the Redbook I (1982) and the Redbook II (1993). The FDA Center for Food Safety and Applied Nutrition (CFSAN) requires a prenatal developmental toxicity study and a multigeneration reproduction study for an adequate safety assessment of food ingredients (Collins et al., 2006; U.S. FDA, 2000a,b).

In the European Union, overall regulation for foodstuffs in human consumption is found in Regulation (EC) 178/2002 [OJ L 31, February 1, 2002] (EC, 2002). Food additives are regulated under Council Directive 89/107/ EEC [OJ L 40, February 11, 1989] on the approximation of the laws of the Member States concerning food additives authorized for use in foodstuffs intended for human consumption (EC, 1989).

\section{Cosmetics}

Although cosmetics are regulated by the FDA in the United States, the legal authority is different from other products regulated by the agency, such as drugs, biologics, and medical devices. With the exception of color additives, cosmetic products and ingredients are

Table 1

Harmonized Guidelines Used in Screening and Testing for Developmental and Reproductive Toxicities for US EPA and OECD

\begin{tabular}{|c|c|c|}
\hline Guideline & US EPA & OECD \\
\hline Prenatal developmental toxicity & OPPTS 870.3700 (U.S. EPA, 1998a) & GL 414 (OECD, 2001a) \\
\hline Reproduction and fertility effects & OPPTS 870.3800 (U.S. EPA, 1998b) & GL 416 (OECD, 2001b) \\
\hline One-generation reproductive toxicity study & $\mathrm{N} / \mathrm{A}$ & GL 415 (OECD, 1983) \\
\hline Reproduction/developmental toxicity screening test & OPPTS 870.3550 (U.S. EPA, 2000a) & GL 421 (OECD, 1995) \\
\hline $\begin{array}{l}\text { Combined repeat dose toxicity study with the } \\
\text { reproduction/developmental screening test }\end{array}$ & OPPTS 870.3650 (U.S. EPA, 2000b) & GL 422 (OECD, 1996) \\
\hline Developmental neurotoxicity & OPPTS 870.6300 (U.S. EPA, 1998c) & GL $426(O E C D, 2007 a)$ \\
\hline
\end{tabular}

OECD, Organisation of Economic Co-operation and Development; OPPTS, Office of Prevention, Pesticides, and Toxic Substances. 
not subject to FDA premarket approval authority. The Office of Cosmetics in CFSAN follows the Redbook guidelines for their required reproductive and developmental studies (U.S. FDA, 2005a). In Europe, the manufacturers have traditionally followed the OECD guidelines.

In Europe, the components of cosmetics are primarily regulated under Council Directive 76/768/EEC [OJ L 262, September 27, 1976] on the approximation of the laws of the Member States relating to cosmetic products and its subsequent amendments (EC, 1976).

Global harmonization for cosmetics regulation is guided by the International Cooperation on Cosmetic Regulation (ICCR) whose members include the United States (FDA/CFSAN), Japan (MHW), the EU (European Commission DG Enterprise and Industry), and Canada (Health Canada) (U.S. FDA, 2010). The purpose of this group is to ensure global consumer protection, and to minimize barriers to international trade.

\section{Consumer Products}

The Consumer Product Safety Commission (CPSC) is an independent federal regulatory agency charged with protecting the public from unreasonable risks from thousands of types of consumer products. The CPSC administers and enforces several laws including the Consumer Product Safety Act, the Consumer Product Safety Improvement Act, and the Federal Hazardous Substances Act (FHSA) (CPSC, 2008a,b,c). In 1992, the Commission finalized the Chronic Hazard Guidelines ( $\mathrm{CHG}$ ), to be used for determining whether customary or reasonably foreseeable handling or use of products subject to the FHSA would present a chronic hazard by containing a carcinogen, neurotoxicant, or reproductive or developmental toxicant (CPSC, 1992). The guidelines also explain certain principles to be used in evaluating the risk resulting from exposure to such products. Although the guidelines are not mandatory and the FHSA does not provide for premarket approval of consumer products, such products subject to the FHSA are expected to be appropriately labeled if they present a chronic hazard, and are subject to enforcement action if they do not carry such a label. No toxicity or exposure testing is required under the FHSA; thus, the CHG are meant to provide general guidance for the evaluation of data by manufacturers and other interested parties for determination of whether a substance is toxic. In practice, CPSC staff scientists consult other guidance in their determinations, which generally undergo external peer review.

Section D of the CHG ( $p$ 46,641-46,644) discusses the evaluation of reproductive and developmental toxicity based on human and animal data. It addresses acceptable risks to children and adults, and discusses definitions of "sufficient" and "limited" evidence of developmental and reproductive toxicity from human and animal studies as well as the categories of "known," "probable," or "possible" developmental and reproductive toxicants.

\section{DIFFERENCES IN REGULATORY PERSPECTIVE ("ONE SIZE DOES NOT FIT ALL")}

Although the traditional developmental toxicity test data that are assessed and incorporated into decisionmaking are virtually identical across the various regulatory organizations, there can be very distinct differences in the way that the data are utilized. The impact of developmental toxicity findings is linked to the mission of each Agency. For example, the EPA utilizes these data to assess potential risks to the human population from exposure to various environmental pollutants, focusing on dose levels that do not result in adverse effects. On the other hand, FDA assesses pharmaceuticals, which generally have some pharmacologic benefit to the target population, and regulatory decision-making for pharmaceuticals must address both the benefits and the risks. Additionally, there are differences in the exposures that must be considered across agencies. For environmental pollutants, exposures are generally inadvertent, whereas for pharmaceuticals, exposures are deliberate. The health and/or susceptibility status of exposed populations may also vary widely. For pharmaceuticals, it is more likely that the health of the individuals being treated has been compromised, whereas that may or may not be the case for those individuals exposed to environmental contaminants. Finally, the developmental toxicity test data under consideration may be used to screen for adverse outcomes and doses, or they may be used for an indepth characterization of the toxicological profile of the chemical.

\section{THE USE OF DEVELOPMENTAL TOXICITY DATA \\ Prioritization and Screening}

Pharmaceuticals. The capabilities for early screening of drug candidates for potential developmental toxicity have evolved tremendously in the last several years and such assays are becoming a regular part of the extensive process of selection of optimal molecules to be advanced for registration and use as medicine. As in all screening processes, the developmental toxicity assays selected for use must have high throughput capabilities and deliver data quickly with precision and reliability to enable differentiation [prioritization] of compounds. Test systems, such as whole embryo culture, zebra fish and stem cell cultures, whose developmental responses are well characterized have the potential to be used in combination with other screening assays to generate data that allow for comparative analysis of toxic potency. These data contribute to an overall evaluation of a drug candidate and may signal deselection of that particular molecule [or class of molecules] from further evaluation. More often, however, the data from early screening alerts a company to a potential developmental risk. In such cases, the subsequent developmental studies can be modified, or focused, or mechanistic studies can be devised, to better assess that potential risk. These assays can aid in compound selection early in the drug development process but they will not replace the more thorough regulatory testing that must occur for a drug to be approved for use [See previous section].

Chemicals. Novel chemicals are typically evaluated in preliminary screening studies. The purposes of screening studies are multiple and often include consideration for early stage product deselection in the event that adverse events are detected in the screening evaluation. Early detection of adverse effects can 
ultimately minimize the numbers of animals used in testing as well as the costs associated with the safety assessment program for a particular chemical. Screening studies can be designed such that several related substances are tested simultaneously with a shared control group. This approach permits comparison of potency among related substances, uses fewer animals by sharing the control group data across the substances, and could ultimately reduce cycle time in the product development process.

Industrial chemicals are used due to their physical chemical properties (e.g. solvent), position within a reaction sequence (e.g. monomer for polymer production), or due to other specific properties crucial to an industrial process (e.g. catalysts). Unlike pesticides or pharmaceutical agents, industrial chemicals are not designed to be biologically active. The level of testing for developmental toxicity endpoints is usually determined by the extent of human exposure, structural alerts, or by the presence of other toxicity characteristics (e.g. mutagenicity). The extent of human exposure for many industrial chemicals is often difficult to quantify so production/import volume is used as a rough predictor of human exposure. Consideration of use and exposure are made for site-restricted industrial intermediates. Chemicals that are produced in large amounts but are primarily restricted to use within industrial plants can be tested in screening studies for developmental endpoints. Examples of these study types are the OPPTS 870.3550/ OECD 421 and OPPTS 870.3650/OECD 422 (Makris, 2010).

Under the recent EU Registration, Evaluation, Authorisation, and Restriction of Chemicals (REACh) legislation, screening studies for developmental toxicity are required under Annex VIII for all substances manufactured or imported in quantities of 10 tonnes or more (Article 12(1)(c)). OECD guideline studies 421 or 422 are required if there is no evidence from structurally related substances, QSAR estimates, or from in vitro methods that the substance may be a developmental toxicant. These studies do not need to be conducted if the chemical is a genotoxic carcinogen or a germ cell mutagen and appropriate risk management measures are implemented. In cases where relevant human exposures can be excluded or if guideline reproductive (OPPTS 870.3800 or OECD 416) and developmental (OPPTS 870.3700 or OECD 414) toxicity studies are available, the screening studies do not need to be performed. If available data indicate that the substance is a reproductive toxicant (Category 1 or 2, R60 C\&L), then testing for developmental toxicity should be considered. If the registrant of the chemical has serious concerns about potential adverse effects on development, the registrant may propose the definitive studies (OPPTS 870.3700 or OECD 414) instead of the screening studies.

\section{Issues That Should be Considered or Addressed in Developmental Toxicity Testing}

Translational medicine, cross-species extrapolation. Ideally, the data obtained to determine the reproductive and developmental toxicity of a compound are derived from animal and /or other nonhuman models before human exposure. Thus, it is critical that the endpoints monitored in our models have relevance for the human condition. These might include metrics of fertility, sexual differentiation and development, etc. The need to relate findings from preclinical models with human outcome suggests that the endpoints used in our models should be validated, i.e. shown to demonstrate some direct correlation with human outcomes. The fact that this has not historically been the case may account for some past failures of animal models to predict adverse human outcome. The lack of detection of the teratogenic effects of thalidomide in animal models is but one example (Schardein, 1998). Future efforts should involve concerted validation efforts, particularly in the case of in vitro and in silico systems, to maximize their utility and predictivity. In the case of whole animal models, it will be critical to utilize the exact same (homologous) endpoints of interest to humans, where possible, or to at least use endpoints (analogues) that correlate with human endpoints or are otherwise predictive. For science to best serve public health needs, data obtained in animal models must be extrapolatable and relevant to humans.

Mode of action data. It is also critical to employ models that metabolize test compounds and exhibit the appropriate pathways and modes of action (e.g. receptor interactions) that are relevant to humans. If, for example, a compound's mechanism/mode of toxic action involves a specific pathway(s) (e.g. oxidative phosphorylation, protein synthesis, apoptosis) or receptor(s) (e.g. glutamate, dopamine, aromatic hydrocarbon), then the model used to screen for toxicity should also share those pathways and/or receptors with humans.

Cumulative exposure issues. In real world situations, humans are often exposed to a given chemical repeatedly and at various life stages. Clearly, acute single exposures to high doses of chemicals can lead to frank poisonings and are of obvious regulatory concern. However, repeated and/or continuous exposures to relatively low doses of compounds or exposures to different chemicals concurrently (i.e. mixtures) or sequentially are often the rules rather than the exceptions and pose more difficult problems for developmental toxicity risk assessment. Experimental designs and approaches to risk assessment should take into consideration the cumulative effects of exposure, many of which can vary depending upon the life cycle stage during which such exposures occur. As a noteworthy example, descriptions of historical testing strategies and an approach to the risk assessment of developmental outcomes resulting from cumulative exposures to multiple phthalate ester chemicals are addressed in a report by the National Research Council (NRC, 2008b).

Critical windows of exposure and effect. In addition to cumulative effects, the life stage at exposure can be extremely critical. There are periods during development in which organisms may be extremely sensitive to chemical exposure. Organs develop at different times and rates and are often exquisitely sensitive to disruption by chemical exposure during this time. Thus, exposure to compounds such as anesthetic agents, alcohol, lead, and other compounds during gestation and/or early postnatal life when organ systems are rapidly developing can have devastating consequences, whereas similar exposures in adults may be relatively innocuous. This issue must continue to figure large in future testing strategies. 
Latency of response. The effects of a toxic insult(s) may not be evident until long after an exposure occurs. For example, treatment of pregnant women with diethylstilbesterol, an estrogen replacement therapy, leads to concomitant fetal exposure in utero. Subsequent cervical cancer developed in some offspring but not until the onset of sexual maturity (Hatch et al., 1998). Efforts to determine the developmental effects of many compounds to which children are exposed (i.e. anesthetics, methyl mercury) have demonstrated clear, primarily functional, adverse effects that become evident as the child develops. Adults exposed to chemicals that destroy the neurotransmitter dopamine can lead to the eventual development of Parkinson's disease, a process that may take years or decades.

Structural vs. functional outcomes. Alterations in the structure of an organism (classic terata, tumors) are relatively easy to find and measure. Changes in function, however, can be much more difficult to detect. This is particularly true for the nervous system since function can be altered sometimes in the absence of detectable structural changes. This necessitates the use of a broad range of assessments or test batteries in efforts to monitor as many functional domains as practicable. It is very clear that animals (and humans) that are physically normal may suffer significant functional abnormalities.

\section{Pharmaceutical Testing and Labeling}

Proposed labeling requirements. Currently, human drug products are assigned a pregnancy category (A, B, C, D, or X) (Kweder, 2008), but there is a proposal (U.S. FDA, 2008) to modify the label requirements in the CFR to eliminate pregnancy categories and put a greater emphasis on the inclusion of human data when available. The Final Rule for the pregnancy and lactation subsections of drug labeling is in the writing and clearance process. Data from animal studies would still be included in the modified drug label, and this initiative would not change the types and amount of animal data that are generally submitted for a drug product.

Pediatric pharmaceuticals. Reproductive toxicity data can be helpful in identifying products that may have different toxicity profiles in adult vs. juvenile populations (U.S. FDA, 2006). These data are used in conjunction with other information (e.g. data from adult animals and humans, knowledge that previously identified target organs of toxicity are undergoing significant growth and development when the drug will be used in children) to determine whether specialized juvenile animal studies are needed for a product. In some circumstances, modified peri/postnatal study designs may be used to fulfill testing needs to explore the potential for a drug's toxicity in juveniles.

\section{Industrial Chemicals and Environmental Pollutants}

Current and future testing of chemicals for developmental toxicity endpoints will be influenced by regulatory and government programs, as these factors control the requirements and prioritization of testing programs.

The testing of industrial chemicals or chemicals that either end up in or are used to produce items common to modern lifestyles is influenced by several ongoing programs. It should be noted that the testing of industrial chemicals for developmental toxicity endpoints is truly a daunting task, as the number of different chemicals used in commerce is in the tens of thousands.

Examples of these programs include the efforts currently ongoing with the OECD Screening Information Data Sets and EPA High Production Volume programs. Both of these programs include evaluation of chemicals for the potential to cause developmental toxicity as a required endpoint. Examples of how this endpoint can be evaluated include data from guideline studies, data from surrogate or related materials, and data from "screening" studies. Typically, however, the endpoint is evaluated using data from a guideline study (OECD Guideline 414, 421 , or 422 , or OPPTS 870.3700 ) in a single species. In certain instances for well-studied materials (typically very high production volume and significant human exposure potential), data are also available for developmental toxicity endpoints collected from multigenerational studies.

Another example of a regulatory program that can lead to testing of industrial chemicals for developmental toxicity endpoints is the $\mathrm{REAcH}$ chemical registration process within the European Union (EU) (EC, 2006), as described above. With the advent of the REACh program within the EU, many additional chemicals will be considered and evaluated for developmental and reproductive toxicity endpoints. It is important to note that the REACh legislation specifically encourages the use of in vitro or alternative (i.e. non-in vivo) test methods when possible, although it is not known to what extent alternative developmental toxicity tests are accepted by the European Chemicals Agency.

There are programs that use developmental toxicity data for labeling or listing of chemicals based on intrinsic hazard information, without regard to issues of exposure or risk. These include the Global Harmonized System (GHS) for classification and labeling (C\&L) and the California Proposition (Prop) 65. Some impacts of these hazard-based systems are described in the following examples.

The use of developmental toxicity testing data in assigning classification and labeling statements to Material Safety Data Sheets (MSDS) and packaging is a universal requirement for all industrial chemicals. The recent adoption of the GHS for classification and labeling has led to specific criteria for evaluating developmental toxicity data for the purposes of preparing MSDS and packaging labels. Although the GHS criteria are open to differing interpretations, it is readily apparent that developmental toxicity data are required to be considered as part of a labeling and classification effort. In addition, many countries are requiring that any GHS decision-making processes conducted by individual companies be available for review by regulatory agencies and the public.

Finally, the end results of developmental toxicity testing can and do lead to chemicals being considered for inclusion on lists of developmental toxicants by regulatory agencies, (e.g. the Prop 65 list in California; CalEPA, 2003). The use of the Prop 65 list in additional regulatory activity (e.g. the California Safe Cosmetics Program; CDPH, 2005) leads to consumer products being surveyed for chemicals with positive developmental toxicity findings, irrespective of exposure or risk levels or route of exposure considerations. For example, 
ethylene glycol is listed as a developmental toxicant by the California Safe Cosmetics Program, based upon an evaluation in a Center for Evaluation of Risks to Human Reproduction (CERHR) monograph (NTP, 2004a). However, the list does not explain that the CERHR monograph limits the concerns for developmental toxicity to high oral dosing regimens and specifically excludes concerns for dermal exposure (an exposure route of significance for the vast majority of cosmetic products). Since inclusion on the Prop 65 list and California Safe Cosmetics Program list invariably affects chemical use and product deselection, it is understandable how concerns with the way developmental toxicity data are used can influence decisions on if, when, and how developmental toxicity testing is conducted.

\section{Agricultural Chemicals}

For agricultural chemicals, screening and testing for developmental and reproductive toxicities are strictly defined in 40 CFR Part 158. Yet, opportunities to utilize new data or new approaches for the assessment of specific issues are evident.

The Food Quality Protection Act (1996) required (among other things) the assessment of inert pesticide ingredients and endocrine disrupting chemicals. Both of these efforts were monumental in scope, and approaches have been developed to address these issues, both of which require an adequate screening level assessment of developmental and reproductive toxicity. For inerts, the use of the OPPTS $870.3550 /$ OECD 421 and the OPPTS 870.3650/OECD 422 screening studies was considered sufficient for a screening level assessment.

For endocrine-disrupting chemicals, a separate screening and testing program was developed in an international collaborative effort. It included defining and validating protocols that could be utilized in a tiered testing scheme, to identify and characterize endocrine disruptive effects. The testing required by this program overlaps some of the current guideline studies (e.g. the twogeneration reproduction study), although it necessitates the collection of even more information. Thus, the development of a replacement protocol is under consideration. Such a protocol was proposed by an ILSIHESI Agricultural Chemical Safety Association effort (Cooper et al., 2006), and this protocol is now undergoing an international discussion as an "extended one generation study" that incorporates the additional endocrine endpoints of concern while reducing study duration and maximizing animal use.

\section{CRITICAL ISSUES AND FUTURE DIRECTIONS}

The future direction of toxicity testing in general has been a topic of intense discussion, as illustrated in a 2007 report by the National Research Council (NRC) described below. A number of specific issues and topics have been raised. These address such diverse issues as new types of exposure and delivery systems (e.g. from nanotechnology), new ways to assess exposure and effects from changes in the genome, transcriptome, proteome, or metabolome, better use (and organization of) existing and new databases/registries, and the development and use of consistent harmonized terminology, so information can be entered, exchanged, extracted, and used across databases, disciplines, and sources.

\section{The Need for a Transformation of the Developmental Toxicology Testing Paradigm}

Currently, regulatory agencies rely on the use of developmental toxicity studies in experimental animal models. The historical path taken has generally been either to make incremental modifications to existing tests (e.g. for the testing of monoclonal antibodies) or to add additional tests to cover endpoints not previously considered (e.g. developmental neurotoxicity). This approach has led over time to a continual increase in the number of tests, cost of testing, use of laboratory animals, and time to develop and review the resulting data. Similarly, the translation and application of such data in risk assessment has been limited by an incomplete understanding of the extent and mechanisms by which chemicals pose hazards to human health. For some substances, such as nanomaterials (i.e. particles of $100 \mathrm{~nm}$ or less in size that are already being used in diverse pharmaceutical, cosmetic, and environmental applications), unique concerns regarding test substance administration and the assessment of effects on reproduction and the developing fetus may need to be addressed in novel ways. This remains an area of preliminary research that may benefit from consideration of a new testing paradigm (NRC, 2008a). Moreover, the application of current risk assessment approaches to meet existing, and evolving, regulatory needs has encountered challenges in accommodating increasingly complex issues (e.g. life-stage sensitivity, mixtures, varying exposure scenarios, cumulative risk, understanding mechanisms of toxicity and their implications in assessing dose-response, and characterization of uncertainty).

Recognizing these limitations in traditional developmental toxicity testing and toxicity testing in general, a report by the NRC on "Toxicity Testing in the 21st Century: a Vision and a Strategy" recommends a landmark transformation in toxicity testing and risk assessment that will focus on toxicity pathways (NRC, 2007b). This approach uses the rapidly evolving scientific understanding of how genes, proteins, and small molecules interact to maintain normal cell function to then determine how some of these interactions can be perturbed by exposure to environmental agents with subsequent events following a toxicity pathway(s) that may eventually lead to adverse health effects. The NRC notes that successful application of new scientific tools in computational, informational, and molecular sciences will inform and produce more credible decision-making with an increased efficiency in design and costs and a reduction in animal usage.

Many regulatory agencies have recognized the need for this transformative shift and have initiated research programs to achieve the vision and goals laid out by the NRC. These include the NIEHS NTP Roadmap for the 21st Century (NTP, 2004b) and the FDA Critical Path Initiative (FDA, 2004; Woodcock and Woolsey, 2008). EPA created the National Center for Computational Toxicology (NCCT) in 2005 and launched the ToxCast ${ }^{\mathrm{TM}}$ research program in 2006 (Dix et al., 2007; U.S. EPA, 2007). The OECD initiated a Molecular Screening for Characterizing 
Individual Chemicals and Chemical Categories Project in 2007, published a monograph on a 2007 Workshop on Integrated Approaches for Testing and Assessment (OECD, 2008b), and actively utilizes Test Guideline Committees and a QSAR Expert Group to ensure global harmonization and validation (OECD, 2005, 2007b) of any new approaches.

\section{Alternative In Vitro and Nonmammalian In Vivo Models for Developmental Toxicity Screening}

The use of alternative in vitro or nonmammalian in vivo models for general or organ/system specific developmental toxicity screening has been a topic of research for the past 30 years. Test systems utilizing cell-, organ-, and embryo-cultures are available (Piersma, 2006) and are used extensively in research. Alternative models that are considered to be validated for regulatory screening by the European Centre for the Validation of Alternative Methods include the embryonic stem cell test, the limb bud micromass, and the rat postimplantation whole embryo culture. In vivo nonmammalian models are also available for developmental toxicity screening. Examples of alternative species that are utilized in morphological and/or functional developmental assessments and/or in mechanistic studies include invertebrates such as the nematode (Caenorhabditis elegans) and fruit fly (Drosophila melanogaster), and vertebrates such as the frog (Xenopus laevis) and zebrafish (Danio rerio) (Lein et al., 2005). It is recognized that data derived from these alternative species can potentially have a critical role in developmental toxicity risk assessment (NRC, 2000).

\section{-Omics Technologies}

-Omics technology is a very exciting new frontier with major initiatives in developmental and reproductive toxicology. The broad term of -omics is used to describe the following technologies:

A. Genomics, which characterizes the genes which are up-regulated (transcribed) or down-regulated, typically immediately after an exposure in a particular tissue, at a particular time, relative to an untreated sample; the body of transcribed mRNAs is termed the "transcriptome";

B. Proteomics, which characterizes the proteins upregulated (increased translation) or downregulated, typically immediately after an exposure in a particular tissue, at a particular time, relative to an untreated sample; the body of translated proteins is termed the "proteome";

C. Metabolomics, which characterizes the profile of endogenous small molecular weight metabolites in a body fluid (serum, urine, amniotic fluid, milk, etc.) or in a supernatant of a homogenate from a particular tissue, body part, embryo, etc., immediately or long after an exposure, relative to an untreated sample; the body of endogenous metabolites present is termed the "metabolome."

These techniques have been used to characterize the early molecular responses of an embryo or fetal organ to chemical exposure through the use of genomics or proteomics, or to characterize the immediate or long term alterations in the profile of endogenous metabolites through the use of metabolomics (e.g. after in vivo or in vitro exposure to an estrogen or antiandrogen). The data can be used to inform mechanism, to distinguish exposed vs. nonexposed subjects or affected vs. unaffected subjects, even long after the exposure has ceased, or to identify subjects with or without a disease or genetic lesion. Such information, especially early in the disease progression or early in the lifetime of the genetically affected individuals, may be important in initiating interventions as soon as possible to improve outcomes.

Pharmaceuticals. Pharmacogenomic data can be used to screen pharmaceutical candidates as part of early research and development. These data are not required to be submitted to FDA, but they may be submitted under the Voluntary Genomic Data Submission program (U.S. FDA, 2005b). The potential for the application of such data in personalized health care has also been recognized, and early initiatives are being implemented (U.S. HHS, 2008).

Environmental agents. The potential uses of -omics data in predictive toxicology and risk assessment for environmental agents, as well as the research and tools that might be needed to implement such a change in paradigm, has been considered in some detail (NRC, 2007a). Applications of this information to hazard screening, the analysis of variation in human susceptibility, the study of mechanisms of action, evaluation of dose-response relationships, cross-species extrapolation, and exposure assessment (including to mixtures) have been discussed in some detail, and there are many on-going research efforts in these areas. The NRC report has specifically recognized an important role for -omics data in characterizing the risk from exposures that occur during development.

\section{Need for Accessibility to Existing Data (Animal and Human)}

It is recognized that as new technologies and bioinformatic methods are utilized, there needs to be a shift toward increased sharing of data. This concept has been raised in relation to -omics data (NRC, 2007a), and there are already a number of research efforts that allow global access to information (e.g. the ILSI-HESI toxicogenomics project and ToxCast ${ }^{\mathrm{TM}}$ ) (ILSI-HESI, 2004; Judson et al., 2009). This concept of data sharing has been incorporated into the REACh program as well, which requires that toxicological data be made publicly available (EC, 2006).

Similarly, human clinical trials must be publicly recorded on a number of websites in the United States and European Union. ClinicalTrials.gov (http:// www.clinicaltrials.gov/), maintained by the NIH, is a registry of clinical trials listing information about a trial's purpose, who may participate, and other information pertinent to the trial conduct. The European Clinical Trials is known as EudraCT (European Union Drug Regulating Authorities Clinical Trials) (http:// www.dkma.dk/1024/visUKLSArtikel.asp?artikelID = 3321) and is a database of all clinical trials in the EU. The EudraCT database was established in May 2004 in accordance with Directive 2001/20/EC. The International Federation of Pharmaceutical Manufacturers and Associations Clinical Trials Portal (http://clinicaltrials. ifpma.org/no_cache/en/myportal/index.htm) is a 
voluntary database that provides information on clinical trials, clinical trial results, and related issues.

Complementary to this issue of globally available data is the need for consistent and universally accepted terminology for characterizing effects. Historically, the developmental toxicology community has embraced this concept, with international collaborative projects and publications on terminology used in the evaluation of fetal specimens (e.g. Wise et al., 1997; Makris et al., 2009). This same attention to consistency and precision in terminology must also be applied to new technologies for developmental toxicity testing.

\section{Importance of Pregnancy Registries}

The FDA Center for Drug Evaluation and Research encourages the establishment of pregnancy registries for products that are likely to be used during pregnancy to treat new or chronic conditions (U.S. FDA, 2002). Pregnancy registries may also be useful for products that are widely used in women of childbearing age, as an inadvertent exposure during pregnancy is common. When concerns regarding a product's potential harm are elevated due to data from animal studies, structureactivity relationships, membership in a pharmacological class known to be reproductive or developmental toxicants, or human case reports, there is a heightened need to establish a pregnancy registry. They can be required as a condition of approval (e.g. a post marketing requirement) for products that are seen as having a potential risk of reproductive or developmental toxicity. Pregnancy registries are important tools for collecting human data to assess the risks and benefits of using a given medication. Data gained through pregnancy registries can be included in drug product labels. Well-designed pregnancy registries enroll women exposed to the drug or drugs of interest, and can evaluate a range of developmental outcomes within a single study and provide estimations of absolute risk for a particular outcome. Such information is needed when counseling women about the risks related to drug exposure. It is important to note, however, that pregnancy registries have limitations. Pregnancy registries generally do not have enough statistical power to evaluate rate outcomes, and unless the sizes of the registries are very large, they are limited in the ability to detect modest increases in risk for a specific malformation. Outcomes that do not manifest until late after birth (such as deficits in behavior or intellectual development) are often not captured.

\section{DISCUSSION/SUMMARY/CONCLUSIONS}

This article presents a broad overview of the use of developmental toxicity testing in the assessment of human health risks associated with exposures to pharmaceuticals (human and veterinary), chemicals (agricultural, industrial, and environmental), food additives, cosmetics, and consumer products. It is evident that developmental toxicology data are utilized extensively to meet the regulatory mandates of a number of United States agencies at the federal and state levels, as well as serving the diverse requirements of multiple international organizations. These data are used for prioritization and screening for pharmaceuticals and chemicals, for evaluating and labeling of pharmaceuticals for adults and children, and for characterizing hazards and risk of exposures to industrial and environmental chemicals (including hazard labeling).

The approaches to developmental toxicity assessment, primarily using standardized protocols that assess apical endpoints in studies that include dose levels that exceed expected relevant human exposures, have existed for many decades. The in vivo study designs utilized in hazard characterization and dose-response assessment for developmental outcomes have not changed substantially over the past 30 years. Now however, there are opportunities to incorporate new technologies and approaches to testing into the existing assessment paradigm, or to apply innovative approaches to various aspects of risk assessment, as recommended by the National Research Council (NRC, 2007b, 2008b, 2009). Efforts are already in place at a number of United States and international regulatory agencies to facilitate the implementation of this concept.

As an expansion of the developmental toxicity testing paradigm occurs, the fact that the data are used for many diverse purposes requires careful consideration. It will be important to maintain the ability to use developmental toxicity data to meet the various critical regulatory needs highlighted in this article. Currently, the common primary use of in vivo developmental toxicity data is in hazard identification or characterization and doseresponse assessment. Opportunities abound to move into new directions, including through the use of in vitro assays, studies conducted in alternative nonmammalian species, the application of new technologies, and in the use of in silico models. In some cases, these new methods will enhance the information already gleaned from in vivo assays. One example might be in the elucidation of mode of action information that may be useful to inform risk assessors about the human relevance of developmental toxicity observed in laboratory animals.

The potential for complete replacement of in vivo animal testing is a lofty and commendable goal that has been established to address ethical principles and in some cases (e.g. REACh), a legislative directive. A significant amount of work needs to be accomplished to translate this concept into reality. For a successful outcome, there needs to be a discernable link established between cross-discipline research planning and implementation and the needs of the risk assessment community. As part of this effort, validation of assays and approaches is critical. Assay reliability, reproducibility, and predictivity will need to be demonstrated through a concerted validation effort. Also of critical importance is the establishment of links between assays and what are considered adverse outcomes in the species of interest; in some cases, it may only be possible to do so in a qualitative (i.e. not quantitative) manner. Realistically, the validation process is not an insignificant task, and it will likely require extensive human, temporal, and capital resources.

In summary, it is evident that there is significant opportunity and promise in the alternative models and new technologies available for the assessment of developmental toxicology for risk assessment. Potential benefits to the current regulatory process include the ability to screen large numbers of chemicals quickly, and with the commitment of fewer resources than traditional toxicology studies, and to refine the risk assessment 
process through a greater understanding of the mechanisms of developmental toxicity and their relevance to potential human risk.

\section{ACKNOWLEDGMENTS}

The authors declare that they have no competing financial interest. We express our appreciation to Patricia Bittner (U.S. Consumer Products Safety Commission) for review and comment. The views expressed in this document are those of the authors and do not necessarily reflect the views or policies of the U.S. Environmental Protection Agency or the U.S. Food and Drug Administration.

\section{REFERENCES}

Brannan K, Fenton S, Hansen DK, et al. 2011. Developmental toxicologynew directions workshop: Refining testing strategies and study designs. Birth Defects Res B Dev Reprod Toxicol (this issue).

CalEPA (California Environmental Protection Agency). 2003. Proposition 65: Safe Drinking Water and Toxic Enforcement Act of 1986 (Amended August 11, 2003). Sacramento, CA: California Environmental Protection Agency, Office of Environmental Health Hazard Assessment. Available at: http://www.oehha.org/prop65.html

Carney EW, Ellis AL, Tyl RW, et al. (2011) Critical evaluation of current developmental toxicity testing strategies: a case of babies and their bathwater. Birth Defects Res B Dev Reprod Toxicol (this issue).

CDPH. 2005. California Safe Cosmetics Program. Sacramento, CA: California Department of Public Health, Occupational Health Branch. Available at: http://www.cdph.ca.gov/programs/cosmetics/ Pages/default.aspx

Collins TFX, Sprando RL, Shackelford ME, et al. 2006. Principles of risk assessment-FDA perspective. In: Hood RD, editor. Developmental and reproductive toxicology-a practical approach. 2nd ed. New York: CRC Press. p 877-909.

Cooper RL, Lamb JC, Barlow SM, et al. 2006. A tiered approach to life stages testing for agricultural chemical safety assessment. Crit Rev Toxicol 36:69-98.

CPSC. 1992. Consumer Product Safety Commission, 16 CFR Part 1500 Labeling Requirements for Art Materials Presenting Chronic Hazards; Guidelines for Determining Chronic Toxicity of Products Subject to the FHSA; Supplementary Definition of "Toxic" under the Federal Hazardous Substances Act: Final Rules. Friday, October 9, 1992. Fed Regist 57:46626-46674.

CPSC. 2008a. Consumer Product Safety Act, amended. 15 U.S.C. $\S \$ 2051-$ 2089. Public Law 92-573; 86 Stat. 1207, October 27, 1972. Consumer Product Safety Commission, Washington, DC. Available at: http:// www.cpsc.gov/about/cpsia/legislation.html

CPSC. 2008b. Consumer Product Safety Improvement Act of 2008. H.R. 4040. Consumer Product Safety Commission, Washington, DC. Available at: http://www.cpsc.gov/about/cpsia/legislation.html

CPSC. 2008c. Federal Hazardous Substances Act, amended, Consumer Product Safety Commission, Washington, DC. Available at: http:// www.cpsc.gov/about/cpsia/legislation.html

CVM. 2006. Guidance for Industry $¥ 3$. General Principles for Evaluating the Safety of Compounds Used in Food-Producing Animals. Rockville, MD: FDA Center for Veterinary Medicine. Available at: http://www.fda.gov/downloads/AnimalVeterinary/GuidanceComplianceEnforcement/GuidanceforIndustry/UCM052180.pdf

Dix DJ, Houck KA, Martin MT, et al. 2007. The ToxCast program for prioritizing toxicity testing of environmental chemicals. Toxicol Sci 95:5-12.

EC (European Communities). 1976. Council Directive 76/768/EEC, OJ L 262, September 27, 1976. p 169.

EC (European Communities). 1989. Council Directive 89/107/EEC, OJ L 40, February 11, 1989. p 27.

EC (European Communities). 2002. Regulation (EC) No 178/2002, OJ L 31, February 1,2002. p 1.

EC (European Communities). 2006. Regulation (EC) No 1907/2006 of the European Parliament and of the Council of 18 December 2006 concerning the Registration, Evaluation, Authorisation and Restriction of Chemicals (REACh). Available at: http://eur-lex.europa.eu/ LexUriServ/LexUriServ.do?uri = CONSLEG:2006R1907:20071123:EN:pdf or: http://ec.europa.eu/environment/chemicals/reach/reach_intro.htm

Hatch EE, Palmer JR, Titus-Ernstoff L, et al. 1998. Cancer risk in women exposed to diethylstilbestrol in utero. J Am Med Assoc 280:630-634.
ICH. 2005. S5(R2). Detection of toxicity to reproduction for medical products \& toxicity to male fertility. ICH Harmonized Tripartite Guildeline. International Committee on Harmonization of Technical Requirements for Registration of Pharmaceuticals for Human Use, Geneva, Switzerland. Available at: http://www.ich.org/cache/compo/276-254-1.html

ILSI-HESI. 2004. Toxicogenomics database. http://www.hesiglobal.org/ Committees/TechnicalCommittees/Genomics/EBI+Toxicogenomics. htm

Judson R, Richard A, Dix DJ, et al. 2009. The toxicity data landscape for environmental chemicals. Environ Health Perspect. 117:685-695.

Knudsen TB, Kavlock RJ, Daston GP, et al. 2011. Developmental toxicity testing for safety assessment: new approaches and technologies. Birth Defects Res B Dev Reprod Biol (this issue).

Kweder S. 2008. Drugs and biologics in pregnancy and breastfeeding: FDA in the $21^{\text {st }}$ century. Birth Defects ResA Clin Mol Teratol 82 (9):605-609.

Lein P, Silbergeld E, Locke P, Goldberg AM. 2005. In vitro and other alternative approaches to developmental neurotoxicity resting (DNT). Environ Toxicol Pharmacol 19:735-744.

Makris SL. 2010. EPA and OECD perspectives on reproductive and developmental toxicity testing. In: Kapp RW, Tyl RW, editor. Reproductive toxicology. 3rd ed. New York: Informa Healthcare. p 86-108.

Makris SL, Solomon H, Clark R, et al. 2009. Terminology of developmental abnormalities in common laboratory mammals (Version 2). Birth Defects Research Part B. 86:227-327.

NRC (National Research Council). 2000. Scientific frontiers in developmental toxicology and risk assessment. Washington, DC: National Academy Press.

NRC (National Research Council). 2007a. Applications of toxicogenomic technologies to predictive toxicology and risk assessment. Washington, DC: The National Academies Press.

NRC (National Research Council). 2007b. Toxicity testing in the 21st century. Washington, DC: The National Academies Press.

NRC (National Research Council). 2008a. Review of federal strategy for nanotechnology-related environmental, health, and safety research. Washington, DC: National Academies Press.

NRC (National Research Council). 2008b. Phthalates and Cumulative Risk Assessment. Washington, DC: The National Academies Press.

NRC (National Research Council). 2009. Science and decisions: advancing risk assessment. Washington, DC: The National Academies Press.

NTP. 2004a. NTP-CERHR Monograph on the Potential Human Reproductive and Developmental Effects of Ethylene Glycol. NIH Publication No. 04-4481. Research Triangle Park, NC: Center for the Evaluation of Risks to Human Reproduction, National Toxicology Program. Available at: http://cerhr.niehs.nih.gov/chemicals/egpg/ ethylene/eg-eval.html

NTP. 2004b. Toxicology in the 21st century: the role of the national toxicology program. Available at: http://ntp.niehs.nih.gov/ntp/ main pages/NTPVision.pdf

OECD. 1983. Test guideline 415. OECD guideline for testing of chemicals. One-generation reproduction toxicity study. Paris, France: Organisation of Economic Co-operation and Development. Available at: http:/ titania.sourceoecd.org/vl $=4717558 / \mathrm{cl}=13 / \mathrm{nw}=1 / \mathrm{rpsv} / \mathrm{cw} /$ vhosts/oecdjournals/1607310x/v1n4/contp1-1.htm

OECD. 1995. Test guideline 421. OECD guideline for testing of chemicals. Reproduction/developmental toxicity screening test. Paris, France: Organisation of Economic Co-operation and Development. Available at: http://titania.sourceoecd.org/vl $=4717558 / \mathrm{cl}=13$ / nw $=1 / \mathrm{rpsv} / \mathrm{cw} / \mathrm{vhosts} /$ oecdjournals/1607310x/v1n4/contp1-1.htm

OECD. 1996. Test guideline 422. OECD guideline for testing of chemicals. Combined repeated dose toxicity study with the reproduction/ developmental toxicity screening test. Paris, France: Organisation of Economic Co-operation and Development. Available at: http:// titania.sourceoecd.org $/ \mathrm{vl}=4717558 / \mathrm{cl}=13 / \mathrm{nw}=1 / \mathrm{rpsv} / \mathrm{cw} /$ vhosts $/$ oecdjournals/1607310x/v1n4/contp1-1.htm

OECD. 2001a. Test guideline 414. OECD guideline for testing of chemicals. Prenatal developmental toxicity study. Paris, France: Organisation of Economic Co-operation and Development. Available at: http://titania.sourceoecd.org/vl $=4717558 / \mathrm{cl}=13 / \mathrm{nw}=1 / \mathrm{rpsv} /$ cw/vhosts/oecdjournals/1607310x/v1n4/contp1-1.htm

OECD. 2001b. Test guideline 416. OECD guideline for testing of chemicals. Two-generation reproduction toxicity study. Paris, France: Organisation of Economic Co-operation and Development. Available at: http:/ titania.sourceoecd.org $/ \mathrm{vl}=4717558 / \mathrm{cl}=13 / \mathrm{nw}=1 / \mathrm{rpsv} /$ $\mathrm{cw} /$ vhosts/oecdjournals/1607310x/v1n4/contp1-1.htm

OECD. 2005. OECD series on testing and assessment, number 34: guidance document on the validation and international acceptance of new or updated test methods for hazard assessment. Organisation for Economic Co-operation and Development. ENV/JM/MONO (2005)14, August18, 2005, Paris. Available at: http://www.oecd.org/ officialdocuments $/$ displaydocumentpdf $/$ cote $=$ env $/ \mathrm{jm} / \mathrm{mono}(2005)$ $14 \&$ doclanguage $=$ en 
OECD. 2007a. Test Guideline 426. OECD Guideline for Testing of Chemicals. Developmental Neurotoxicity Study. Paris, France: Organisation of Economic Co-operation and Development. Available at: http: / / titania.sourceoecd.org $/ \mathrm{vl}=4717558 / \mathrm{cl}=13 / \mathrm{nw}=1 /$ $\mathrm{rpsv} / \mathrm{cw} /$ vhosts/oecdjournals/1607310x/v1n4/contp1-1.htm

OECD. 2007b. Series on testing and assessment no. 69, guidance document on the validation of (quantitative) structure-activity relationship (Q)SAR models. Organisation for Economic Co-operation and Development. ENV/JM/MONO(2007)2, February15, 2007, Paris. Available at: http://www.oecd.org/dataoecd/55/35/ 38130292.pdf

OECD. 2008a. OECD Series on testing and assessment, number 43: guidance document on mammalian reproductive toxicity testing and assessment. Organisation for Economic Co-operation and Development. ENV/JM/MONO(2008)16, July 24, 2008, Paris. Available at: http:/ / www.olis.oecd.org / olis / 2008doc.nsf / linkto / env-jm-mono (2008) 16

OECD. 2008b. Series on testing and assessment no. 88, workshop on integrated approaches to testing and assessment. Organisation for Economic Co-operation and Development. ENV/JM/ MONO(2008)10, May 27, 2008, Paris. Available at: http://www.oecd.org/dataoecd/45/52/40705314.pdf

Piersma A. 2006. Alternative methods for developmental toxicity testing. Basic Clin Pharmacol Toxicol 98:427-431.

Schardein JL. 1998. Animal/human concordance. In: Slikker W, Chang LW, editors. Handbook of developmental neurotoxicology. San Diego: Academic Press. p 687-708.

Shuey D, Kim JH. Introduction-developmental toxicology-new directions. Birth Defects Res B Dev Reprod Biol (this issue).

Tyl RW. 2010. FDA and ICH perspectives on reproductive and developmental toxicity. In: Kapp RW, Tyl RW, editors. Reproductive toxicology. 3rd ed. New York: Informa Healthcare. p 68-85.

U.S. EPA (U.S. Environmental Protection Agency). 1991. Guidelines for Developmental Toxicity Risk Assessment. Office of Research and Development, Risk Assessment Forum, EPA/600/FR-91/001. Fed Regist 56:63798-63826.

U.S. EPA. 1998a. OPPTS 870.3700, Prenatal Developmental Toxicity Study, Health Effects Test Guidelines, EPA 712-C-98-207, Washington, DC. Available at: http://www.epa.gov/ocspp/pubs/frs/publications/ Test_Guidelines/series870.htm

U.S. EPA. 1998b. OPPTS 870.3800, Reproduction and Fertility Effects, Health Effects Test Guidelines, EPA 712-C-98-208, Washington, DC. Available at: http://www.epa.gov/ocspp/pubs/frs/publications/ Test_Guidelines/series870.htm

U.S. EPA. 1998c. OPPTS 870.6300, Developmental Neurotoxicity Study, Health Effects Test Guidelines, EPA 712-C-98-239, Washington, DC. Available at: http://www.epa.gov/ocspp/pubs/frs/publications/ Test_Guidelines/series870.htm

U.S. EPA. 2000a. OPPTS 870.3550, Combined Repeated Dose Toxicity Study with the Reproduction/Developmental Toxicity Screening Test, Health Effects Test Guidelines, EPA 712-C-00-367, Washington, DC. Available at: http://www.epa.gov/ocspp/pubs/frs/publications/Test_Guidelines/series870.htm

U.S. EPA. 2000b. OPPTS 870.3650, Reproduction/Developmental Toxicity Screening Test, Health Effects Test Guidelines, EPA 712-C-00-368, Washington, DC. Available at: http://www.epa.gov/ocspp/pubs/ frs/publications/Test_Guidelines/series870.htm

U.S. EPA. 2007. ToxCast ${ }^{\mathrm{TM}}$ Program. Available at: http://www.epa.gov/ ncct/toxcast/

U.S. FDA. 2000a. Redbook 2000. Toxicological Principles for the Safety Assessment of Food Ingredients. IV.C.9.a. Guidelines for Reproduction Studies. College Park, MD: Center for Food Safety and Applied
Nutrition, U.S. Food and Drug Administration. Available at: http:// www.cfsan.fda.gov/ $\sim$ redbook/redivc9a.html

U.S. FDA. 2000b. Redbook 2000. Toxicological Principles for the Safety Assessment of Food Ingredients. IV.C.9.b. Guidelines for Developmental Toxicity Studies. College Park, MD: Center for Food Safety and Applied Nutrition, U.S. Food and Drug Administration. Available at: http://www.cfsan.fda.gov/ redbook/redivc9b.html

U.S. FDA. 2002. Guidance for industry: establishing pregnancy exposure registries. Rockville, MD: Center for Drug Evaluation and Research, Center for Biologics Evaluation and Research, U.S. Food and Drug Administration. Availabl at: http://www.fda.gov/cder/guidance/ 3626fnl.htm

U.S. FDA. 2004. Challenge and opportunity on the critical path to new medical products. Rockville, MD: U.S. Department of Health and Human Services. U.S. Food and Drug Administration. Availabe at: http: / / www.fda.gov /downloads/ScienceResearch/SpecialTopics / CriticalPathInitiative/CriticalPathOpportunitiesReports/ucm11411.pdf

U.S. FDA. 2005a. FDA authority over cosmetics. College Park, MD: CFSAN Office of Cosmetics and Colors. Availabe at: http:// www.cfsan.fda.gov/ dms/cos-206.html

U.S. FDA. 2005b. Guidance for Industry: Pharmacogenomic Data Submissions. Rockville, MD: Center for Drug Evaluation and Research, U.S. Food and Drug Administration, Center for Biologics Evaluation and Research, Center for Devices and Radiological Health, U.S. Food and Drug Administration. Available at: http:// www.fda.gov/cder/guidance/6400fnl.pdf

U.S. FDA. 2006. Guidance for industry: nonclinical safety evaluation of pediatric drug products. Rockville, MD: Center for Drug Evaluation and Research, U.S. Food and Drug Administration. Available at: http://www.fda.gov/cder/guidance/5671fnl.pdf

U.S. FDA. 2008. Content and format of labeling for human prescription drug and biological products; requirements for pregnancy and lactation labeling, proposed rule, May 29, 2008. Fed Regist 73: 30831-30868.

U.S. FDA. 2010. International Cooperation on Cosmetic Regulation (ICCR). Rockville, MD: International Programs/Harmonization Initiatives. Available at: http://www.fda.gov/InternationalPrograms/HarmonizationInitiatives/ucm114513.htm

U.S. HHS (U.S. Department of Health and Human Services). 2008. Personalized health care: pioneers, partnerships, progress. Initiative on personalized health care. Office of the Assistant Secretary for Planning and Evaluation, Department of Health and Human Services, Washington, DC. Available at: http://www.hhs.gov/ myhealthcare/news/phc_2008_report.pdf

VICH. 2004a. Studies to evaluate the safety of residues of veterinary drugs in human food: reproductive toxicity testing (VICH GL22). Brussels: International Cooperation on Harmonisation of Technical Requirements for Registration of Veterinary Medical Products. http://www.vichsec.org/en/guidelines2.htm

VICH. 2004b. Studies to evaluate the safety of residues of veterinary drugs in human food: developmental toxicity testing (VICH GL32). Brussels: International Cooperation on Harmonisation of Technical Requirements for Registration of Veterinary Medical Products. Available at: http://www.vichsec.org/en/guidelines2.htm

VICH. 2008. Target animal safety for veterinary pharmaceutical products (VICH GL43). Brussels: International Cooperation on Harmonisation of Technical Requirements for Registration of Veterinary Medical Products. Available at: http://www.vichsec.org/en/guidelines2.htm

Wise LD, Beck SL, Beltrame D, et al. 1997. Terminology of developmental abnormalities in common laboratory mammals (version 1). Teratology 55:249-292.

Woodcock J, Woolsey R. 2008. The FDA critical path initiative and its influence on new drug development. Annu Rev Med 59:1-12. 